\title{
Annotationes Epigraphicae XII \\ Zu einigen Inschriften aus den römischen Provinzen Germania inferior und Germania superior*
}

\author{
Andreas Kakoschke
}

I.

Seit der Mitte des 18. Jahrhunderts ist ein Steinensemble aus Frankfurt-Heddernheim/Nida bekannt, das aus einer $107 \mathrm{~cm}$ hohen Geniusstatue und einer zugehörigen Basis (Höhe $54 \mathrm{~cm}$ - Breite 50,5 cm - Tiefe 34,5 cm) aus Sandstein besteht (Abb.1). Die im Sockel eingeschlagene Inschrift datiert das Objekt durch die Angabe der amtierenden Konsuln in das Jahr 230 n. Chr.

Vor einigen Jahren hat HERZ die vielsagende Inschrift in einem Aufsatz ausführlich besprochen und hinsichtlich ihrer sozialen, rechtlichen und psychologischen Implikationen eingehend interpretiert. Dabei gibt HERz den Text, der ihm zufolge „im wesentlichen als unproblematisch" gelten darf, sinngemäß wie folgt wieder:"

\author{
In $\bullet h($ onorem $) \cdot d($ omus $) \cdot d($ ivinae $)$ \\ genium plateae novi vi- \\ ci cum edicula(!) et ara

Aufgrund des erwähnten Aufsatzes von HERz lässt sich zu der Inschrift an sich kaum etwas Neues beitragen. Überraschenderweise enthält die von HERZ dargebotene Um-

\footnotetext{
* Bei den Herausgebern der FeRA, Herrn Prof. Dr. K. Matijević (Flensburg) und Herrn Dr. P. Probst (Hamburg), bedanke ich mich wie immer für einige wichtige Hinweise. Für die Bereitstellung der Photos danke ich Herrn Prof. Dr. R. Wiegels und Herrn Prof. Dr. K. Matijević (CIL XIII/2-Projekt, Universitäten Trier/Flensburg) sowie Herrn Dr. O. Harl (Universität Wien). Für kritische Hinweise danke ich meinen Kollegen Herrn Dr. St. Oelschig (Osnabrück) und Herrn Prof. Dr. St. F. Pfahl (Düsseldorf). - Ligaturen bzw. Nexus werden im Folgenden durch unterstrichene Buchstaben wiedergegeben. - Die bisherigen Annotationes Epigraphicae erschienen in den vorhergehenden Ausgaben der FeRA. Die Annotationes Epigraphicae III finden sich im GFA 19 (2015), 179-197.

${ }^{1}$ Herz 1989, 159. Taf.30. Abb.1-2 (Photos). In der Umschrift wurden Worttrenner und Ligaturen gemäß den Angaben im CIL und der Photographie (Abb.1) hinzugefügt. Zudem hat der Autor kleinere Fehler bei der Wiedergabe des Textes [Zeile 5, Alexand(rianae); Zeile 11, c(ivis) R(omanus)] stillschweigend korrigiert, ein Ausrufezeichen in Zeile 3 ergänzt, die antike Rasur in Zeile 5 kenntlich gemacht, das $\mathrm{O}$ in Zeile 9 und 12 wie eingeschlagen mit einem Überstrich wiedergegeben und das $\mathrm{T} z \mathrm{zu}$ T(itus) (Zeile 4) bzw. T(iti) (Zeile 8) aufgelöst. - Der Stein befindet sich heute im Stadtmuseum Wiesbaden (Sammlung Nassauischer Altertümer, Inv.-Nr.341).
} 
schrift jedoch einen kleinen Fehler bzw. eine Ungenauigkeit, die alle folgenden Bearbeiter der Inschrift übernommen haben und die sich auch schon in älteren Textwiedergaben findet.

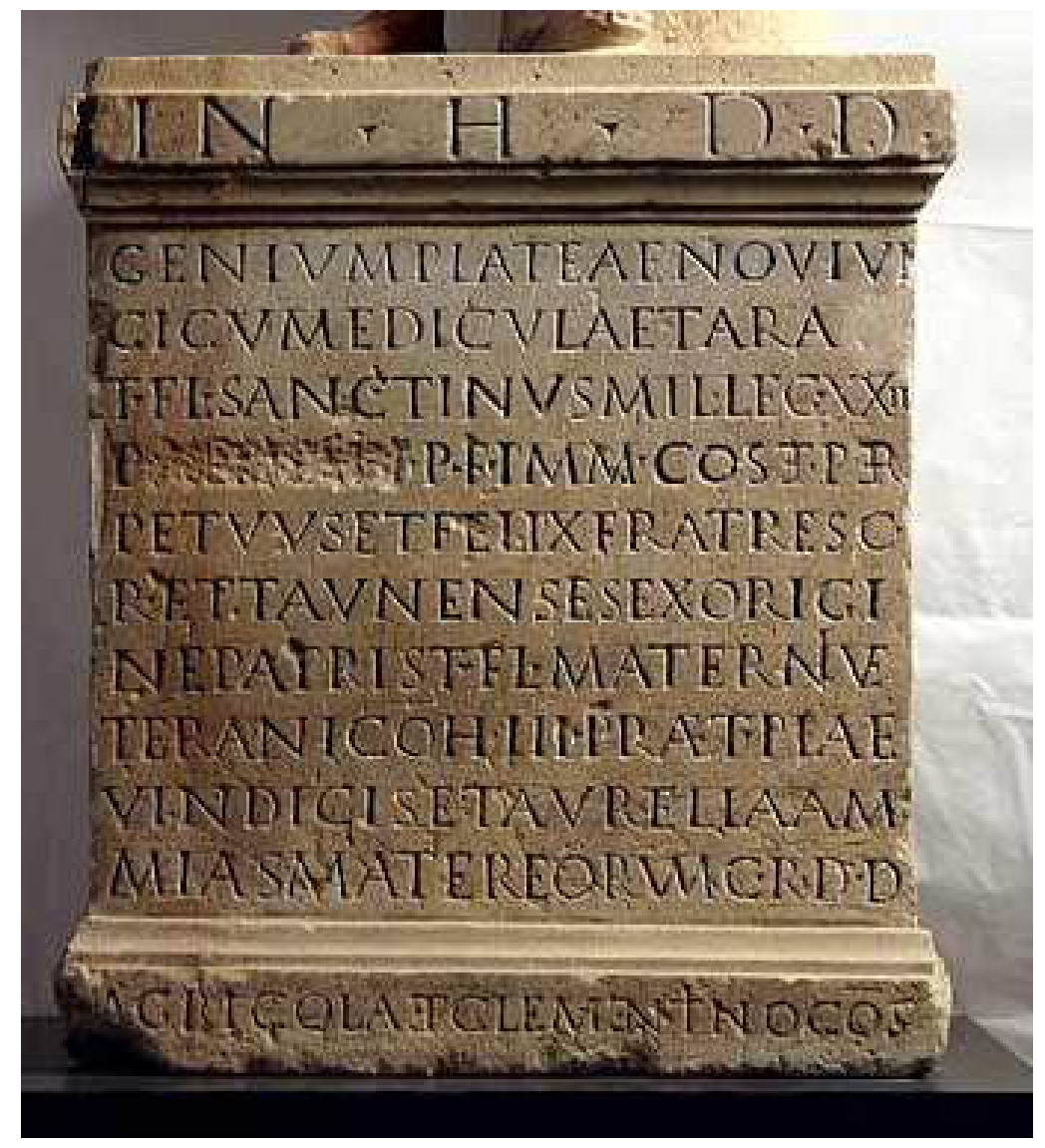

Abb.1: Weihinschrift aus Frankfurt-Heddernheim/Nida: CIL XIII 7335 [C Ortolf Harl, Wien (LUPA)]

M.E. muss es in Zeile 4 entgegen den bisherigen Lesungen nicht T(itus) Fl(avius), sondern T(iti) Flavii) heißen. ${ }^{2}$ Schließlich bezieht sich T FL nicht nur auf den zuerst genannten Sohn, auf Sanctinus, sondern ebenso auf Perpetuus und Felix. ${ }^{3}$ Gleichermaßen beziehen sich die nachfolgenden Angaben (fratres cives Romani et Taunenses ex origine patris ...) auf alle drei Söhne. Daher spricht HERZ in seinen Ausführungen ganz zu Recht von den „Titi Flavii““. ${ }^{4}$ In der beigefügten Übersetzung heißt es jedoch „Titus Flavius Sanctinus ... und (seine) Brüder Perpetuus und Felix“.5 Es muss jedoch heißen T(iti) Fl(avii) Sanctinus ... Perpetuus et Felix fratres, also „Die Titi Flavii

\footnotetext{
${ }^{2}$ Vgl. dagegen u.a. die Lesungen von EsPÉRANDIEU 1931, 77-78. Nr.111 (mit Photo), SCHOPPA² 1965, 17. Nr.33, Schillinger-HÄFEle 1982, 60-61. Nr.23 (mit Photo), MEIER-ARENDT 1983, 46. Nr.19 (mit Photo), FreIS² 1994, 228. Nr.141, SPICKERMANN 1994, 256. Nr.4, B. GESEMANN, SJ 49 (1998), 95 (mit Photo) und KAKoschKe 2002, 257-258. Nr.2.39 sowie die Angaben in den Datenbanken LUPA (Nr.7111), EDCS (Nr.11001427) und EDH (Nr.HD041967).

3 S. auch KaKoschKe 2006, 184. GN 497-101, KAKOSCHKE 2008, 191. CN 2338-4 [T. Fl(avius) Perpetuus] und KAKOSCHKE 2006, 183. GN 497-46, KAKOSCHKE 2007, 345. CN 1288-6 [T. Fl(avius) Felix].

${ }^{4}$ HeRz 1989, 160, 162.

${ }^{5}$ Herz 1989, 160, 159. - Vgl. z.B. auch die Übersetzung bei MEIER-ARENDT 1983, 46. Nr.19 („,.. sowie seine Brüder Perpetuus und Felix ...").
} 
Sanctinus ... Perpetuus und Felix, die Brüder ...“. Das von Herz in der Übersetzung eingefügte ,seine“ (eius), das im lateinischen Text fehlt, ist somit nicht notwendig.

Entsprechende Namensformen mit einem Gentiliz im Plural und mehreren nachfolgenden Cognomina sind natürlich mehrfach bezeugt - auch in den germanischen Provinzen. ${ }^{6}$ Seltener sind Belege mit einem vorangestellten Pränomen und einem Gentiliz, jeweils im Plural. Aber auch für diese Form lassen sich Nachweise (aus den germanischen Provinzen) erbringen. ${ }^{7}$ In der vorliegenden Inschrift folgt auf das erste Cognomen ein längerer Einschub, der den Status des Sanctinus näher bezeichnet: mil(es) leg(ionis) XXII P(rimigeniae) ... Einen vergleichbaren Einschub weist auch eine Inschrift aus Frankfurt-Heddernheim/Nida auf. Dort heißt es: cum Stephaniis Maximo dec(urio) c(ivitatis) s(upra) s(crip)tae et Festa Maximino Maximina Honorata filiis. ${ }^{8}$ Entsprechend liest man auf einer auch von HERZ (unvollständig) zitierten Inschrift aus Mainz/Mogontiacum: Victori(i) Ursus frum(entarius) et Lupus filii. ${ }^{9}$ In diesem Sinne ist auch in der vorliegenden Inschrift mit Sicherheit T(iti) Fl(avii) zu lesen. ${ }^{10}$

Die Lesung entspricht ganz dem von HERz deutlich gemachten Anliegen der drei Brüder. Wie HeRz betont, möchten die Brüder ja ganz bewusst darauf hinweisen, dass das Bürgerrecht in ihrer Familie bis in die Zeit der flavischen Kaiser zurückreicht. Die Familie will sich damit klar von den zeitgenössischen Neubürgern abheben, die das römische Bürgerrecht erst im Zuge der constitutio Antoniniana erhielten. Insofern ist es aus inhaltlichen Gründen undenkbar, dass sich in der kollektiven Weihinschrift, in der auch der Vater der Brüder mit vollem Namen genannt wird [T(itus) Fl(avius) Maternus, Zeile 8], die entscheidende Angabe T FL (in Zeile 4) nur auf einen der drei Brüder bezieht.

\section{II.}

Im Jahre 1843 oder 1844 übermittelte der Oberlehrer DiLlenBuRgeR dem in Bonn ansässigen „Verein von Alterthumsfreunden im Rheinland“ die Lesung einer heute verlorenen römerzeitlichen Inschrift, die man kurz zuvor in einem Grab in TitzBettenhoven (Kr. Düren) entdeckt hatte. Die Lesung des klassischen Philologen, der am Gymnasium in Aachen Latein und Griechisch unterrichtete, wurde im „Jahrbuch des Vereins von Altertumsfreunden im Rheinland" in Majuskelschrift wie folgt wiedergegeben: ${ }^{11}$

\footnotetext{
${ }^{6}$ Verwiesen sei beispielhaft auf folgende Fälle aus den germanischen Provinzen: CIL XIII 6436 [Dreieichenhain; Liberalini(i) Iuvenis et Iuventina et Maternus et Faustus et Ibernalis fili(i)], 6458 [Großbottwar; Longini(i) Pacatus Martinula Hilaritas Speratianus fili(i)], 7298 [MainzKastel/Castellum Mattiacorum; Servandi(i) Eternus Servanda Severina Barbar(a)], 1. N. 75. Nr.227 [Bingen/Bingium; Privati(i) Secundinus et Tertinus et Confinis], 2. N. 115. Nr.241 [Braunkohlengrube Wachtberg I bei Frechen-Benzelrath; Viponi(i) Vitalis Lellua Candidus Fervesa Quintus].

${ }^{7}$ CIL XIII 7821 (Wollersheim; [..] Primini(i) [Res]pectus [et] Ianuarius), 8571 (Meerbusch-OssumBösinghoven; Q(uinti) Iul(ii) Quietus et [I]ucundus et Ursulus). - Von Interesse sind in diesem Zusammenhang u.a. auch folgende Inschriften: CIL XIII 7119 [Mainz/Mogontiacum; L(ucii) et C(aii) et Sex(ti) Valeriorum ...], 8161 [Hürth-Gleuel; Sexti Val(erius) Peregrin(us) et Val(erius) Felicio], 3. N. 202-203. Nr.222 [Köln/CCAA; C(aii) et M(arci) Versulatium ...].

${ }^{8}$ CIL XIII $7352=$ MEIER-ARENDT 1983, 54-55. Nr.28 (mit Photo) $=$ EDH Nr.HD036527.

${ }^{9}$ CIL XIII $11810=$ KAKOSChKE 2002, 131-132. Nr.1.107 = EDH Nr.HD032982. Vgl. HeRZ 1989, 162.

${ }^{10}$ Der Auftraggeber bzw. der Steinmetz hätten die Lesung durch ein doppeltes T (als Zeichen für den Plural) eindeutiger gestalten können. Diese „Lesehilf““ wurde jedoch offenbar nicht in Erwägung gezogen.

${ }^{11}$ L. LeRsCH, BJb 4 (1844), 182-183. Nr.80. - Die Majuskelwiedergabe DilLEnBURGERs findet sich später auch bei STEINER 1851, 183. Nr.1220, BRAMBACH 1867, 135. Nr. 618 und im CIL (XIII 7894).
} 


\title{
MATRONIS GAVADIABVS CALDIV - SEVI RVS $\cdot$ E $\cdot$ SVPER $\mathbf{L} \mathbf{M}$
}

\begin{abstract}
Abb.2: Weihinschrift aus Titz-Bettenhoven: CIL XIII 7894
\end{abstract} [nach L. LERSCH, BJb 4 (1844), 182-183. Nr.80]

$\mathrm{Zu}$ dieser Textwiedergabe bot LERSCH, der die Inschrift nicht aus eigener Anschauung kannte, folgende Umschrift: Matronis Gavadiabus Caldiu(s) Severus e(t) Sperus (sic) lubenste merito. ${ }^{12}$ Obwohl die Inschrift LERSCH zufolge auf einem ,noch gut erhaltene(n) Stein“ stand, dessen Größe er mit „2 F. 4 Z. lang, 1 F. 5 Z. breit, 7 Z. dick" (Höhe 76,80 cm - Breite 39,51 cm - Tiefe 2,59 cm) angibt, zweifelte LERSCH die Lesung DillenbURGERs offenbar an. So vermerkte er zu dem von DillenBURGER in Zeile 4 gelesenen E SVPER: „Es liesse sich auch et Superinius oder ein ähnlicher Name lesen; oder stand vielleicht EXS. IMPER. die gewöhnliche Formel auf diesem Steine?" Die Textwiedergabe DILLENBURGERs war - wie weiter unten zu zeigen ist wahrscheinlich fehlerhaft. ${ }^{13}$ Allerdings ist die Lesung der vierten Zeile wohl mehr oder weniger als korrekt zu bezeichnen. Hier stand höchstwahrscheinlich ET SVPER, wobei E und T wohl ligiert waren. Die den Matronae Gavadiae geweihte Inschrift wurde also von einem Severus (oder Sevirus) und einem Super gesetzt.

Fraglich ist jedoch die Deutung und Lesung des offenbar abgekürzten Gentilnomens CALDIV in Zeile 3. BRAMBACH gibt den Namen (laut Index) mit Caldiu(s) wieder. Denselben Namen bieten RIESE, GUTENBRUNNER und RösGER. ${ }^{14}$ Der Name könnte von dem einmal in der Narbonensis belegten lateinischen Cognomen Caldus abgeleitet sein. ${ }^{15}$ WeISGERBER vertritt die Lesung Caldiv(---) bzw. (laut Index) Caldiv(ius). ${ }^{16}$ Der Lesung Caldiv(ius) schloss sich u.a. auch die EDCS an. ${ }^{17}$ Sowohl Caldius als auch Caldivius sind allerdings nicht weiter bezeugt. ${ }^{18}$ Gegen die Lesung Caldiu(s) oder Caldi(vius) spricht zudem die Tatsache, dass die folgenden zwei Cognomina eine Pluralform fordern. Schließlich wird es sich bei Sevirus und Super

\footnotetext{
${ }^{12}$ L. LERSCH, BJb 4 (1844), 182-183. Nr.80.

${ }^{13}$ Grund zu dieser Annahme liefert auch die Inschrift CIL XIII 7895 (= EDH Nr.HD076820) aus TitzBettenhoven, die zusammen mit der Inschrift CIL XIII 7894 entdeckt wurde. DILLENBURGER sandte eine Lesung des Textes in Majuskelschrift nach Bonn, die L. LERSCH, BJb 4 (1844), 182. Nr.79 zusammen mit folgender Umschrift publizierte: Matronis Ettr(ai)enis ... He(re)n(ius?) solvit votum lubens merito. Da der Stein nicht verloren ging, lässt sich mit Bestimmtheit sagen, dass die Lesung DILLENBURGERs äußerst unzuverlässig war. Auf dem Stein steht nämlich folgender Text: Matronis / Ett $\left\{t_{\text {\} }}\right.$ ra/henis / et / Gesa/henis / M(arcus) Iul(ius) Amandus. DILLENBURGER übermittelte eine Inschrift mit sechs, statt sieben Zeilen und gab z.B. statt des Dedikantennamens in Zeile 7 (M IVL AMANDVS) die Buchstabengruppe VITVLM(!) an.

${ }^{14}$ Brambach 1867, 370, RIESE 1914, 329. Nr. 3143, GUTENBRUNNER 1936, 215. Nr.56/1, RÖSGER 1996, 13. - Zu Caldius s. Whatmough 1949, 955, OPEL II 22 (mit dem vorliegenden Beleg), KAKOSCHKE 2006, 117. GN 242 (mit dem vorliegenden Beleg). Bei Schulze 1966 und SOLIN/SALOMIES ${ }^{2} 1994$ ist der Name nicht verzeichnet.

${ }^{15}$ CIL XII 979 (Les Beaux). S. auch OPEL II 22 (mit falscher CIL-Nr.).

16 Weisgerber 1968, 27. Nr.119. - Zu Caldivius s. Weisgerber 1968, 74, 146, 147, 160, 201, 401, 402, 434, WEISGERBER 1969, 428, KUHN 1978, 395 (germanisch?), REICHERT 1987-1990, Teil I, 166 (,nicht G“), KAKOSCHKE 2006, 117. GN 242a (mit dem vorliegenden Beleg).

${ }^{17}$ EDCS Nr.11100119 [„Caldiv(ius) Sev $<e=I>r u s\{E\}$ Super"]. S. auch RAEPSAET-ChARLIER 2011, 220.

${ }^{18}$ Vgl. Solin/SALOMIES² 42 [mit dem Beleg „CALDIV(IVS) CIL XIII 7894“].
} 
um Brüder oder um Vater und Sohn gehandelt haben, die denselben Familiennamen führten. ${ }^{19}$

Im gesamten Inschriftenmaterial des Imperium Romanum finden sich nur drei Gentilnomina, die mit der Buchstabengruppe CALD beginnen. Sieht man von den Namen Caldonius und Caldurnius ab, die durch eine Ehreninschrift der cohors $V$ vigilum aus Rom bezeugt sind ${ }^{20}$ verbleibt nur noch ein Name: Caldinius. Das Gentiliz tritt fast ausschließlich in der Germania inferior auf, genauer in Köln/CCAA und im Hinterland der $C C A A .^{21}$ Aus diesem geographischen Bereich stammt auch die von DiLLENBURGER überlieferte Inschrift. Die Feststellung führt zu der Vermutung, dass die Inschrift aus Titz-Bettenhoven den Namen CALDINI bot. ${ }^{22}$ Am Ende des Namens stand möglicherweise eine NI-Ligatur. Vielleicht war das $\mathrm{N}$ auch mit dem vorangehenden und dem folgenden I verbunden, so dass am Ende ein $\mathrm{N}$ mit zwei weiter nach oben gezogenen vertikalen Hasten eingeschlagen war. ${ }^{23}$

Wie der vermeintliche Lesefehler DiLlenburgers zu Stande kam, bleibt offen. Gleiches gilt für die weiteren vermeintlichen Lesefehler der Matronen-Inschrift (SEVIRVS statt SEVERVS ${ }^{24}$ E statt ET bzw. ET in Ligatur). Vermutlich war der Text des Steins aufgrund von Beschädigungen doch nicht problemlos zu entziffern. Leider könnte nur ein wiederentdeckter Stein oder eine vertrauenswürdige Abschrift oder Zeichnung die hier geäußerten Überlegungen hinsichlich der korrekten Lesung der Inschrift bestätigen oder widerlegen.

III.

Im ca. $30 \mathrm{~km}$ nordwestlich von Köln gelegenen Morken-Harff (Rhein-Erft-Kreis) entdeckte man im Jahre 1958 zahlreiche Weihesteine für die bis dahin unbekannten Matronae Austriahenae. Sämtliche Matronensteine, die vermutlich alle aus einem in der Nähe gelegenen Matronenheiligtum stammen, wurden 1960 von KOLBE publiziert. ${ }^{25}$ Einige Steine sind lediglich fragmentarisch erhalten, so dass sich der Inschriftentext nicht weiter ergänzen ließ. Zu diesen Fragmenten zählt ein Altar aus gelbem Sandstein mit verzierten Seitenflächen (Abb.3), bestehend aus drei Teilen, von denen zwei (mit Buchstabenresten) aneinanderpassen (Höhe $26 \mathrm{~cm}$ - Breite 21,5 $\mathrm{cm}$ - Tiefe $22 \mathrm{~cm}$; Höhe $49 \mathrm{~cm}$ - Breite $24 \mathrm{~cm}$ - Tiefe $22 \mathrm{~cm}$ ). KolBE gibt die In-

\footnotetext{
19 Vergleichbare Matronenweihungen sind selbstverständlich bekannt: CIL XIII 7938 [ZülpichRövenich; Suetoni(i) Certus et Paternus], 2. N. 94. Nr. 145 [Bonn/Bonna; Iuli(i) Romulus et Peregrinus], Kolbe 1960, 81. Nr.51 (Morken-Harff; Pompe[i(i)] Maternus et P[---]) u.a.

${ }^{20}$ CIL VI $31234=$ EDR Nr.104184.

${ }^{21} \mathrm{Zu}$ Caldinius s. KAKOSCHKE 2006, 116-117. GN 241 (mit sechs Belegen für Niedergermanien). Hinzu kommt: CIL XIII 7847 = A. KAKOschKE, ZPE 197 (2016), 241-242. Nr.2. Abb.2-3 (Photos) (Merzenich).

${ }^{22}$ Entsprechende Vermutungen finden sich schon bei M. IHM, BJb 83 (1887), 150. Nr.304 und P. JOERRES, BJb 100 (1896), 115. Nr.6.

${ }^{23}$ Eine entsprechende Ligatur lag wohl ferner in der verschollenen Weihinschrift CIL XIII 7878 (= BRAMBACH 1867, 133. Nr.603 = EDH Nr.HD075097) aus Linnich-Tetz vor. Die ersten Editoren des Textes, der Pfarrer von Tetz, W. J. MuCKENHEIM, und der Steuereinnehmer J. P. BüTtGEN, interpretierten diese INI-Ligatur jedoch fehlerhaft als ein größeres $\mathrm{N}$ [GRATNVS VICTOR ET GRATN(A)E ALANIS $\leq \mathrm{E}>\mathrm{T}$ MNA(!)]. In dieser Fassung fand die Inschrift auch Eingang ins CIL. Zu Näherem s. A. KAKOSCHKE, FeRA 27 (2015), 24-26. Nr.3.

${ }^{24}$ Zum Cognomen Sevirus s. jedoch WeISGERBER 1968, 97, 160, 201, 206 [„,Die Schreibung Sevirus 7894 wird man wohl bei der Gruppe Severus belassen, doch ist es kaum ein Steinmetzversehen, da (abgesehen von dem möglichen Mitspielen eines einheimischen Elementes s. o. S. 130) sowohl die Möglichkeit eines Anklanges von sevir wie eine lautliche Annäherung von $e$ an $i$ die Abweichung begründen kann.“"], OPEL IV 78 und KAKOSCHKE 2008, 332-333. Nr.2839 (mit den Belegen CIL XIII 7894, ILGN Nr.185).

${ }^{25}$ KolBE 1960, 50-124.
} 
schrift des kombinierten Fragments (ohne Ligaturen und Worttrenner) wie folgt wieder: ${ }^{26}$

\author{
[Austri]ahenis \\ $\left[.{ }^{3-4} ..\right]$ nanus \\ [Can]didus ex \\ [im(perio)] ip(sarum) l(ibens)
}

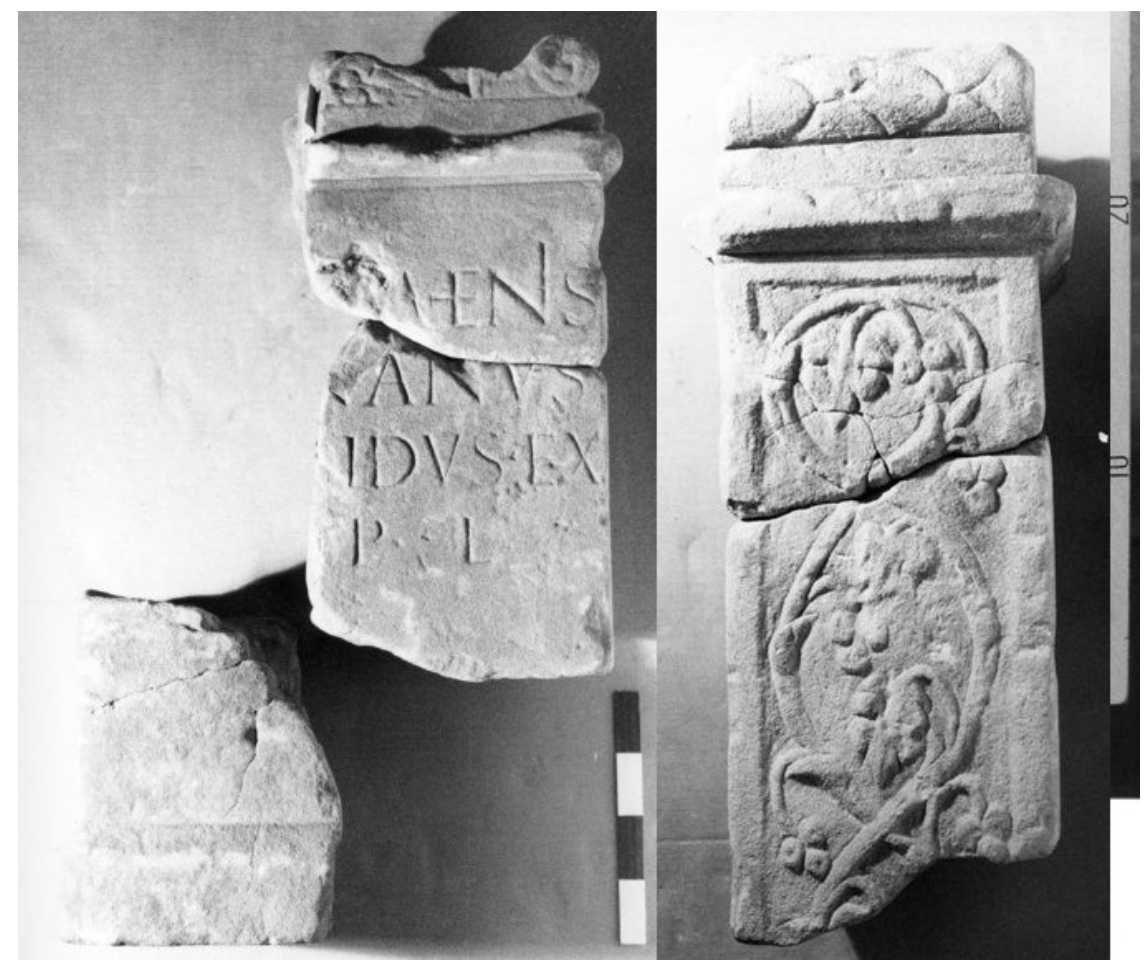

Abb.3: Weihinschrift aus Morken-Harff: KOLBE 1960, 64. Nr.20

(C) CIL XIII/2-Projekt, Universitäten Trier/Flensburg)

Zeile 1, mit dem Matronenbeinamen, und Zeile 3, mit dem Cognomen des Dedikanten, lassen sich recht sicher ergänzen, ${ }^{27}$ zumal auch die Breite des Altars aufgrund der noch zur Hälfte erhaltenen Teile des Giebels rekonstruierbar ist (Abb.4). Hinsichtlich der zweiten Zeile bemerkt KOLBE im Kommentar: „In Z. 2 ist der Anfang vom Gentile, eventuell auch das Pränomen, weggebrochen." Ein auf -nanus endendes Gentiliz ist allerdings nicht bekannt, so dass KOLBE keinen Ergänzungsvorschlag anbieten kann. Es erscheint jedoch auch sehr unwahrscheinlich, dass das Gentiliz des Dedikanten recht untypisch auf -nanus endete. Näher liegend wäre die Lesung der Buchstabengruppe NANIVS. Allerdings lässt sich über der rechten Vertikalen des zweiten $\mathrm{N}$ eindeutig kein Rest eines ligierten I erkennen. Den Weg zu einer stimmigen Deutung des fragmentarisch erhaltenen Namens weist wohl ein ebenfalls in Morken-Harff bezeugter Dedikant namens L. Laubasnianus Ammalenus. ${ }^{28}$ Höchswahrscheinlich trug auch der Mann der vorliegenden Weihung ein einheimisches

\footnotetext{
${ }^{26}$ KolBe 1960, 64. Nr.20. Taf.17 (Photo). Vgl. auch die KolBE folgende Lesung in der EDH (Nr.HD080763). - Der Stein befindet sich heute im Rheinischen Landesmuseum in Bonn (Inv.Nr.58,701).

${ }^{27}$ Eine alternative Ergänzung der Buchstabengruppe DIDVS bietet sich nicht an. - Zum gängigen Candidus, das in Niedergermanien recht häufig auftritt, s. OPEL II 30-31, KAKOSCHKE 2007, 200-201. $\mathrm{CN} 644$.

28 3. N. 205-206. Nr.233 = EDH Nr.HD080378.
} 
Pseudogentiliz auf -ianus. Diese Namen treten fast ausschließlich im Hinterland der $C C A A$ auf. Insgesamt sind aus dem ubisch geprägten Raum an die 20 dieser Namen bekannt. ${ }^{29}$ Auf dem Stein stand also wahrscheinlich NIANVS mit einer nicht mehr vorhandenen NI-Ligatur. Wie die noch erhaltenen Buchstaben des Matronenbeinamens zeigen, hat der Steinmetz mit Ligaturen gearbeitet. Eine weitere Ligatur in Zeile 2 wäre also keineswegs ungewöhnlich. ${ }^{30}$

Von den bisher bekannten Namen auf -ianus bietet sich aufgrund der noch zu lesenden Buchstaben und der Breite des Steins nur der Name Sabinianus für eine Ergänzung an (Abb.4). Das vom lateinischen Namen Sabinus abgeleitete Pseudogentiliz findet sich u.a. in Bad Münstereifel-Iversheim (Kr. Euskirchen). ${ }^{31}$ Geht man davon aus, dass der Name in der vorliegenden Inschrift mit einer INI-Ligatur eingeschlagen wurde, stand das Gentiliz zentriert in der Zeile, mit einem kleinen Freiraum rechts und links des Namens. Allerdings ist diese Ergänzung selbstverständlich nicht sicher, da weitere bisher nicht bezeugte, aber theoretisch denkbare -ianus-Namen, wie *Albinianus, *Dignianus, *Latinianus oder *Rufinianus, die Lücke gefüllt haben können.

Angesichts der rekonstruierten Breite des Altars kann die Lesung KolBEs noch in zwei weiteren Punkten leicht verbessert werden. So darf man mit einiger Berechtigung davon ausgehen, dass im Matronenbeinamen die Buchstaben $\mathrm{A}$ und $\mathrm{V}$, eventuell die Buchstaben $T$ und $R$, ligiert waren. Da AV-Ligaturen am Anfang des Beinamens in Morken-Harff recht häufig auftreten, ${ }^{32}$ wird man vielleicht eher eine AV-Ligatur erwarten. Zudem hat der Steinmetz in der letzten Zeile wahrscheinlich nicht die Buchstaben IM IP L, sondern I IP L eingeschlagen. ${ }^{33}$ Wie die folgende Rekonstruktionszeichnung verdeutlicht (Abb.4), steht die noch stärker abgekürzte Schlussformel, im Gegensatz zu der von KOLBE favorisierten Buchstabengruppe, zentriert auf dem Stein. Eine vergleichbare, zentrierte Anordnung der Buchstaben lässt sich auch auf anderen Steinen aus Morken-Harff beobachten. ${ }^{34}$

Zusammenfassend kann somit folgende geringfügig verbesserte Neulesung des Textes vorgeschlagen werden:

\author{
[Austri]ahenis \\ [-3-4-]n[i]anus \\ [Can]didus $\bullet$ ex \\ $[$ i(mperio) $\bullet$ ip(sarum) $\bullet l($ ibens $)$
}

\footnotetext{
${ }^{29}$ A. KAKOSChKE, FeRA 38 (2019), 5-24 (mit sämtlichen Belegen und einer Verteilungskarte).

${ }^{30}$ Am Rande sei vermerkt, dass der zuvor erwähnte Weihestein des L. Laubasnianus Ammalenus aus Morken-Harff ebenfalls eine NI-Ligatur im Gentiliz bietet.

${ }^{31}$ CIL XIII 7943 = EDH Nr.HD077537. - Zum Namen s. OPEL IV 40, KAKOSCHKE 2006, 341. GN 1077 (mit allen Belegen).

${ }^{32}$ Kolbe 1960, 55. Nr.2. Abb.3 (Zeichnung), 61. Nr.8. Taf.13 (Photo), 65. Nr.23. Taf.18 (Photo), 6869. Nr.28. Abb.13 (Zeichnung), 69-70. Nr.29. Abb.14 (Zeichnung), 70-71. Nr.31. Abb.16 (Zeichnung), 73-74. Nr.34. Taf.20 (Photo), 82. Nr.55. Taf.23 (Photo), 87. Nr.66. Taf.18 (Photo), 87-88. Nr.70. Abb.28 (Zeichnung), 89. Nr.72. Abb.30 (Zeichnung), 95. Nr.86. Abb.33 (Zeichnung).

33 Vergleichbare stark abgekürzte Schlusswendungen bieten auch folgende Weihinschriften aus Morken-Harff: KolBE 1960, 70. Nr.30. Abb.15 (Zeichnung), 72-73. Nr.33. Abb.18 (Zeichnung), 77. Nr.40. Abb.22 (Zeichnung), 87. Nr.69. Abb.27 (Zeichnung), 98. Nr.97. Abb.35 (Zeichnung).

${ }^{34}$ Kolbe 1960, 55. Nr.2. Abb.3 (Zeichnung), 55-56. Nr.3. Abb.4 (Zeichnung), 58-59. Nr.6. Abb.7 (Zeichnung), 66. Nr.25. Taf.17 (Photo), 66-67. Nr.26. Taf.18 (Photo) (= AE 2014, 912), 69-70. Nr.29. Abb.14 (Zeichnung), 70. Nr.30. Abb.15 (Zeichnung), 88-89. Nr.71. Abb.29 (Zeichnung), 98. Nr.97. Abb.35 (Zeichnung).
} 


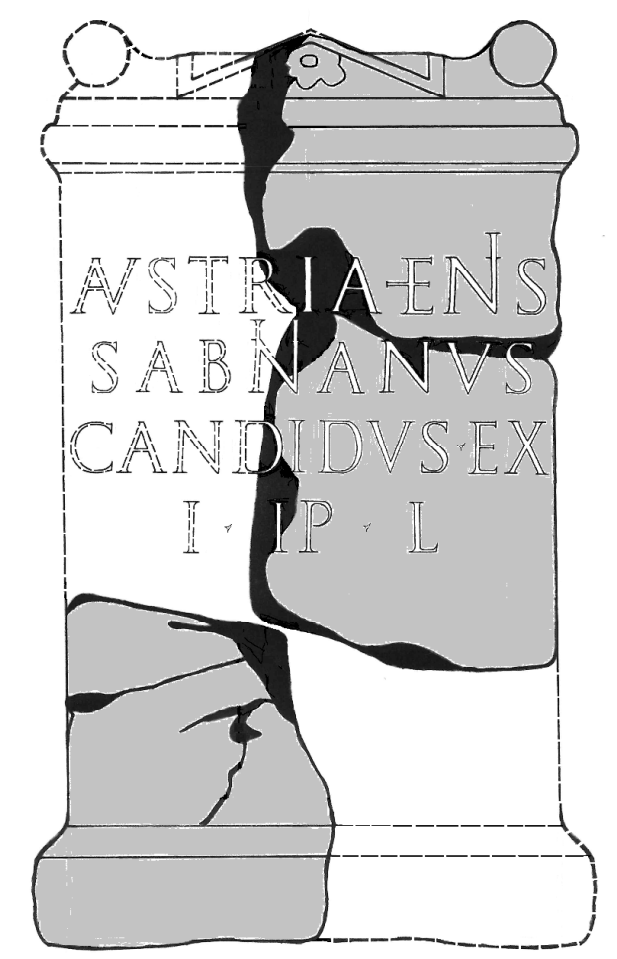

Abb.4: Leicht idealisierte Rekonstruktionszeichnung der Weihinschrift KOLBE 1960, 64. Nr.20 aus Morken-Harff (mit beispielhafter Ergänzung der Zeile 2) (Zeichnung A.K.)

IV.

Vor einigen Jahrzehnten publizierte STUART die Funde aus einem römischen Gräberfeld in Nijmegen/Noviomagus (Provinz Gelderland). Der an der Nordwestseite des römischen Legionslagers gelegene Begräbnisplatz wurde laut STUART das ganze erste Jahrhundert n. Chr. hindurch genutzt, vor allem in claudischer und flavischer Zeit. ${ }^{35}$ $\mathrm{Zu}$ den vorgestellten Objekten des Areals zählt auch ein fragmentarisch erhaltener glattwandiger Becher mit einer eingeritzten Besitzerinschrift (Abb.5). Der Text steht auf der Wand des Bechers, gleich über dem Fuß. STUART gibt für das Graffito, das keine Aufnahme in der L'Année Épigraphique fand, folgende Lesung: C VANERI OPTATI. ${ }^{36}$

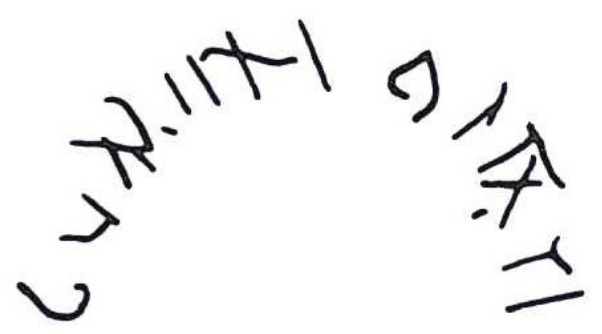

Abb.5: Graffito aus Nijmegen/Noviomagus: STUART 1977, 71. Nr.50 (aus: STUART 1977, 148. Abb.60/50)

\footnotetext{
${ }^{35}$ STUART $1977,71-73,75$.

${ }^{36}$ StUART 1977, 71. Nr.50, 148. Abb.60/50 (Zeichnung). Das Fragment findet sich bei StUART 1977, 139. Abb.51/8 (Zeichnung). - Das Becherfragment lagert heute im Museum Het Valkhof in Nijmegen.
} 
Wie viele andere Besitzerinschriften von Gebrauchskeramik nennt der Text also eine Person im genitivus possessivus, in diesem Fall eine Person mit tria nomina, also eine Person mit gehobener Rechtsstellung. Das unscheinbare Graffito ist insofern interessant, als es ein bisher nicht bezeugtes Gentiliz bietet, den Namen Vanerius. Die Nomenklatur des Besitzers und die Zeitstellung des Objekts deuten dabei mit Sicherheit auf ein italisches Gentiliz.

Die Lesung STUARTs ist allerdings nicht zweifelsfrei. Denn bei näherer Betrachtung scheint es sich bei der von STUART gelesenen AN-Ligatur eher um eine ALLigatur zu handeln. Dabei zeigt die horizontale Haste des L aufgrund der Rundung des Bechers nach oben. $\mathrm{Zu}$ lesen ist demnach das überaus verbreitete (und eher nichts sagende) Gentiliz Valerius. ${ }^{37}$

\section{V.}

Aus Saverne/Tres Tabernae (dép. Bas-Rhin) stammt ein fragmentarisch erhaltener hausförmiger Grabstein (Höhe $71 \mathrm{~cm}$ - Breite $30 \mathrm{~cm}$ - Tiefe $46 \mathrm{~cm}$ ) aus grauem Sandstein, den man zwar schon im Jahre 1929 entdeckte, der aber erst vor kurzem wissenschaftlich publiziert wurde (Abb.6). Der in das fortgeschrittene zweite oder dritte Jahrhundert zu datierende Grabstein fand sich beim Bau des „,nouveau collège“ in der Rue Poincaré Nr.8. GoubET gibt die Inschrift des Steins, die auf einem vertieften Schriftfeld steht, wie folgt wieder: ${ }^{38}$

$$
\begin{array}{ll} 
& \text { D(is) }[M(\text { anibus })] \\
& \text { Crati[ae] } \\
& \text { Amani- } \\
& \text { ae Cara- } \\
& \text { tus mar(itus) } \\
& \text { p(onendum) } c \text { (uravit) }
\end{array}
$$

Die Lesung wurde auch von der L'Année Épigraphique sowie den elektronischen Datenbanken EDH und LUPA übernommen. ${ }^{39}$ Dabei bemerkt die L'Année Épigraphique jedoch im Kommentar zur Inschrift, dass die Lesung des Namens Amania in Zeile 3/4 unsicher ist.

Der Name des Gatten, Caratus (Zeile 4/5), bereitet bei der Lesung kaum Schwierigkeiten, wenngleich das $\mathrm{T}$ in dem eingeschlagenen Namen sehr einem I ähnelt. Die Lesung Caraius kann allerdings ausgeschlossen werden, da das keltische Caratus mehrfach bezeugt ist und die meisten Belege für diesen Namen ebenfalls aus Saverne/Tres Tabernae stammen. ${ }^{40}$

Die Namen der verstorbenen Frau sind laut WEISS bisher nicht weiter bezeugt. ${ }^{41}$ Gemäß WeISS lässt sich das Gentiliz Cratia möglicherweise mit dem

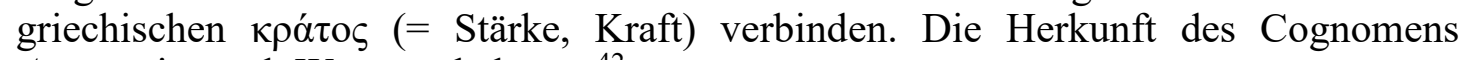
Amania ist nach WeISS unbekannt. ${ }^{42}$

\footnotetext{
${ }^{37}$ Zum Namen s. OPEL IV 142-146, KAKOSCHKE 2006, 410-415. GN 1346.

${ }^{38}$ Goubet 2015, 104. Nr.18 (mit Photo). - Der Stein gehört heute zur Sammlung des Musée du chateau des Rohan in Saverne (Inv.-Nr. Bh 10).

${ }^{39}$ AE 2015, 997, EDH Nr.HD079210, LUPA Nr.27925.

${ }^{40}$ Zum Namen s. OPEL II 36, KAKOSCHKE 2007, 212. CN 690 (mit Belegen).

${ }^{41}$ N. WeISS, in: Goubet 2015, 60. S. jedoch einen Beleg für das Cognomen Cratia in Oberitalien: PAIS 1888, Nr.992 (Ventimiglia). Und die Inschrift CIL XIII 17930 (Timgad/Thamugadi) aus Numidien bietet den Namen Amania Sextulla Sex(ti) ' $f$ '(ilia).

${ }^{42}$ N. WeISS, in: Goubet 2015, 60.
} 


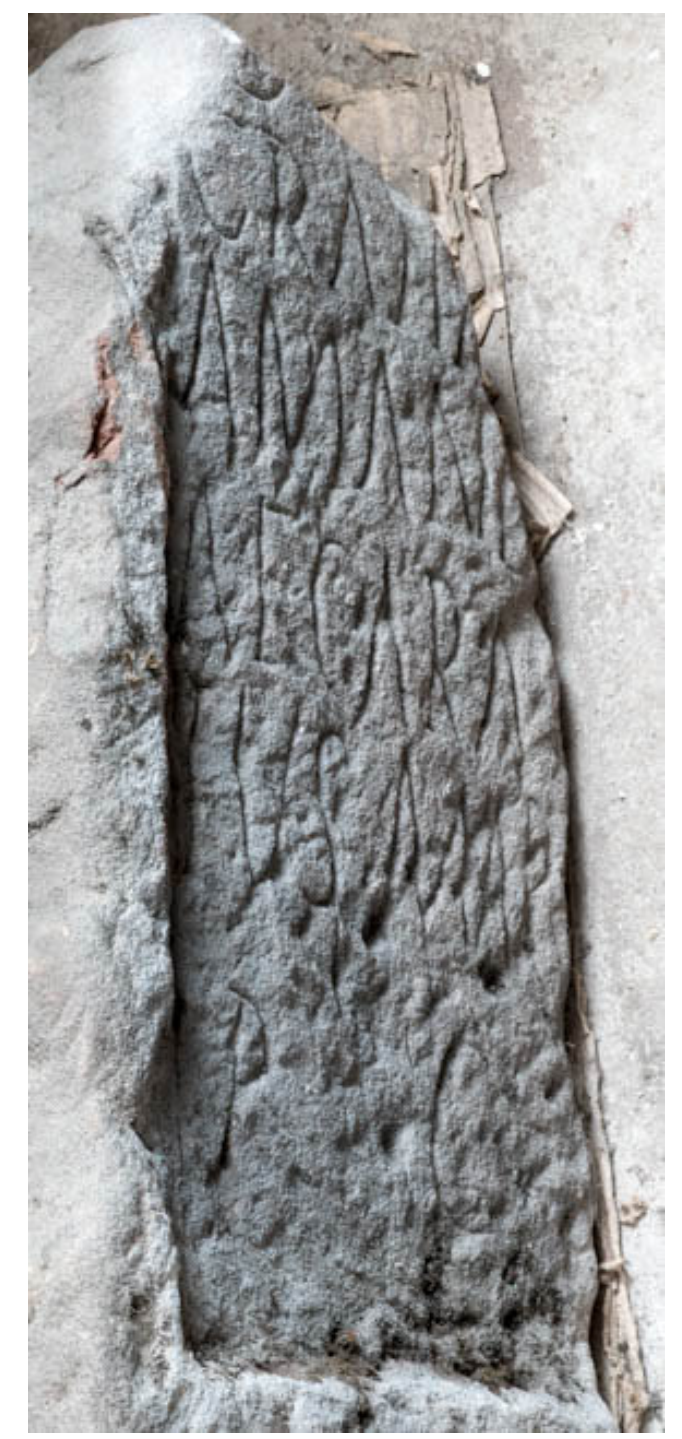

Abb.6: Grabinschrift aus Saverne/Tres Tabernae: AE 2015, 997

[C Musées du chateau des Rohan (Photo: Ortolf Harl)]

M.E. lässt sich statt des ungewöhnlichen Namens Cratia Amania ebenso der gängige Name Gratia Amanda oder auch 'G'ratia Aman'd'a entziffern. In dem vermeintlichen $\mathrm{C}$ am Beginn von Zeile 2 darf man wohl ein $\mathrm{G}$ erblicken, zumal sich der Buchstabe im unteren Bereich mit seiner nach oben auslaufenden Haste von dem in Zeile 4 und 6 eingeschlagenen schmalen $\mathrm{C}$ unterscheidet. Der letzte Buchstabe in Zeile 3 war möglicherweise kein I, sondern ein D. Offenbar steht die Vertikale genau am Ende des Schriftfeldes. Der Bogen des D wurde daher eventuell auf den Rand geschlagen. Im unteren Bereich des Buchstabens ist allem Anschein nach ein Rest des Bogens zu erkennen. Für die vorgeschlagene Lesung spricht, wie oben schon angedeutet, auch die Tatsache, dass sowohl das vom lateinischen Namen Gratus abgeleitete Pseudogentiliz Gratius als auch das Cognomen Amandus recht weit verbreitet waren. ${ }^{43}$

Am Rande sei noch erwähnt, dass in dem Namen des Dedikanten, Caratus,

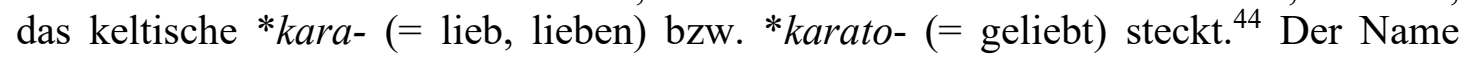

\footnotetext{
${ }^{43} \mathrm{Zu}$ Gratius s. OPEL II 171, KAKOSCHKE 2006, 199. GN 557 (mit Belegen). Zu Amandus s. OPEL I ${ }^{2}$ 45-46, KAKOSCHKE 2007, 88-89. CN 146 (mit Belegen).

${ }^{44}$ Delamarre 2007, 215.
} 
weist somit eine weitgehende semantische Übereinstimmung mit dem lateinischen Namen der Frau, Amanda (amandus = liebenswürdig, lieblich), auf.

\section{VI.}

Im Gebiet der Lingonen, in Dijon/Dibio (dép. Côte-d'Or), in der Rue de Gray, fand sich im Jahre 1867 ein fragmentarischer Grabstein aus Kalkstein (Abb.7), ein „pyramidion“ bzw. „obélisque lingon“ (Höhe $32 \mathrm{~cm}$ - Breite $20 \mathrm{~cm}$ - Tiefe 16,5 cm). LE BOHEC gibt den kurzen Text des Steins aus der Zeit zwischen 50 und $200 \mathrm{n}$. Chr. wie folgt wieder: ${ }^{45}$

$$
\begin{aligned}
& \text { Balat- } \\
& \text { ulla } \\
& \text { Matu- } \\
& \text { cisi (filia) }
\end{aligned}
$$

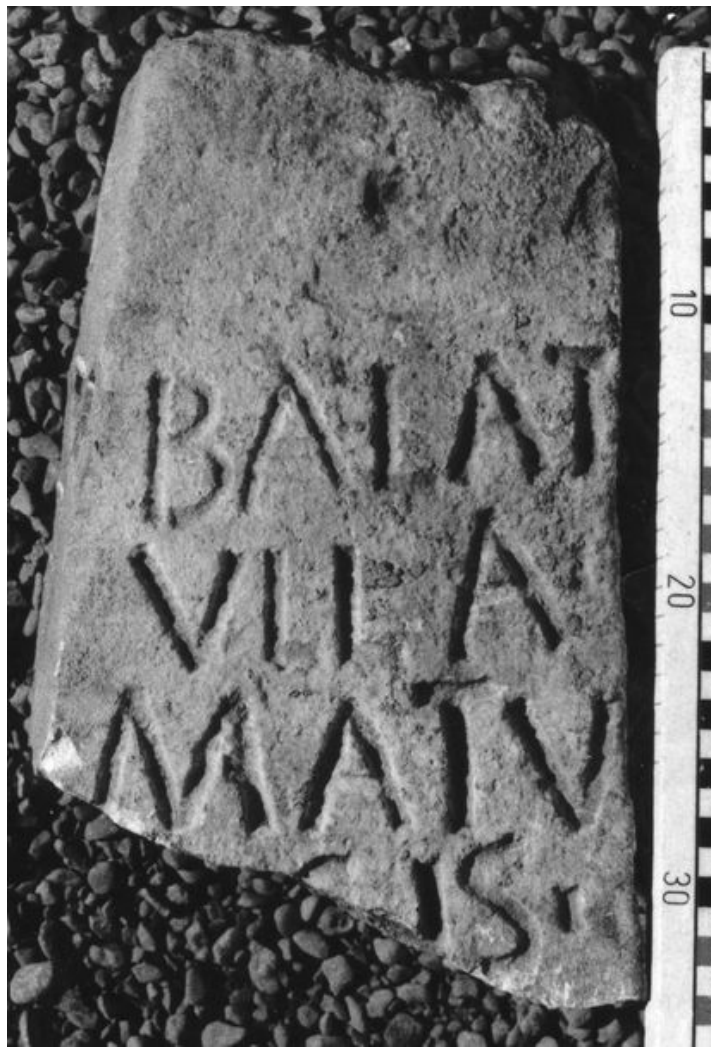

Abb.7: Grabinschrift aus Dijon/Dibio: CIL XIII 5496/97 (C CIL XIII/2-Projekt, Universitäten Trier/Flensburg)

Im Gegensatz zu den Angaben im CIL und bei JOUBEAUX ${ }^{46}$ entziffert LE BOHEC am Ende von Zeile 4 ein I und somit die Buchstabengruppe CISI. Bei dem vermeintlichen I am Ende der Zeile handelt es sich jedoch lediglich um einen länglichen Worttrenner. Ein entsprechender Worttrenner findet sich ebenso am Ende von Zeile 2. Diese etwas

\footnotetext{
45 LE BOHEC 2003, 77. Abb.36 (Photo). S. auch die LE BOHEC folgende Lesung in der EDCS (Nr.10800904). - Der Stein befindet sich heute im Musée archéologique de Dijon (Inv.-Nr.232).

${ }^{46}$ CIL XIII 5496/97, JoubEAUX 1989, 234-235. Nr.36 (mit Photo).
} 
ungewöhnlichen Wort- bzw. Silbentrenner zieren z.B. auch den Grabstein der Atticilla aus Dijon/Dibio. ${ }^{47}$

Ferner ignoriert LE BOHEC das Fehlen von mindestens zwei Buchstaben am Beginn von Zeile 4. JoubeAuX liest daher völlig zu Recht den Namen ,Matu/[...]is““. Allerdings erkennt er nicht den oberen Rest eines $\mathrm{C}$ am Beginn der fragmentarisch erhaltenen Zeile. Es könnte sich hier auch um den Rest eines G handeln. Ein S - wie JOUBEAUX vermutet - liegt wohl nicht vor, wie die Ausformung des folgenden $\mathrm{S}$ in der Zeile zeigt. ${ }^{48}$

Die Lesung des nicht weiter bezeugten Namens Matucisus bzw. Matucisi durch LE BOHEC kann somit nicht zutreffen. ${ }^{49}$ JOUBEAUX schlägt keine Ergänzung vor, verweist aber auf den möglicherweise verwandten Namen Maturcus, ${ }^{50}$ der sich auf einer Inschrift aus Nuits-Saint-Georges in der Gallia Lugdunensis, im Gebiet der Häduer, findet. ${ }^{51}$

Da im nicht allzu weit entfernten Saverne/Tres Tabernae ein Mann namens Carantillus Maturicis fil(ius) bezeugt ist, ${ }^{52}$ sei hier für die Inschrift aus Dijon/Dibio angesichts fehlender Alternativen mit einiger Berechtigung die Lesung des keltischen Namens Maturix bzw. Maturicis vorgeschlagen. ${ }^{53}$ Von dem fehlenden I vor der Buchstabengruppe CIS ist dabei im oberen Bereich nichts mehr zu erkennen, da es wahrscheinlich wie das vorhandene I nach dem $C$ etwas kleiner eingeschlagen wurde.

Die Grabinschrift aus Dijon/Dibio, deren unterer Teil verloren ist, lässt sich also vielleicht wie folgt lesen:

$$
\begin{aligned}
& \text { Balat- } \\
& \text { ulla } \bullet \\
& \text { Matu- } \\
& \text { [ri?]cis (filia) }
\end{aligned}
$$

\section{VII.}

Ebenfalls aus Dijon/Dibio (dép. Côte-d'Or) stammt eine fragmentarische Grabinschrift (Höhe $33 \mathrm{~cm}$ - Breite $55 \mathrm{~cm}$ - Tiefe $25 \mathrm{~cm}$ ) aus Kalkstein mit Darstellung des Verstorbenen in einer Rundnische (Abb.8). Der Grabstein wurde im Jahre $1830 \mathrm{im}$ Flussbett der Suzon, im Bereich der Westnekropole, nahe dem alten botanischen Garten entdeckt. LE BOHEC, der die Inschrift in das zweite Jahrhundert n. Chr. datiert, gibt den kurzen Text des Steins wie folgt wieder: ${ }^{54}$

\section{Marcianus Iovi(i) [f(ilius)] $D$ (iis) [M(anibus)]}

\footnotetext{
${ }^{47}$ CIL XIII $5493=$ LE BOHEC 2003, 75 . Abb.34 (Photo).

48 JOUBEAUX 1989, 234-235. Nr.36 (mit Photo).

${ }^{49} \mathrm{Vgl}$. auch die Angaben bei DelamarRe 2007, 129, der den Beleg aus Dijon/Dibio unter dem Lemma Matucisius führt. Das OPEL III 66 hat den Namen dagegen in der Form Matucus aufgenommen. KAKOSCHKE 2008, 103. CN 1988 listet den Namen unter dem Lemma Matux auf.

50 JOUBEAUX 1989, 235.

${ }^{51}$ CIL XIII 2846.

52 1. N. 48-49. 148/150 = GouBET 2015, 159. Nr.93 (mit Photo) = EDH Nr.HD025648.

${ }^{53}$ Zum Namen s. OPEL III 67, DelamARre 2007, 129, 226, 230, KAKOSCHKE 2008, 101. CN 1984 (jeweils mit dem Beleg aus Saverne/Tres Tabernae).

${ }^{54}$ LE BOHEC 2003, 93-94. Nr.120. Abb.49 (Photo). Vgl. auch die entsprechende Lesung in der EDCS (Nr.10800942). - Der Stein befindet sich heute im Musée archéologique de Dijon.
} 


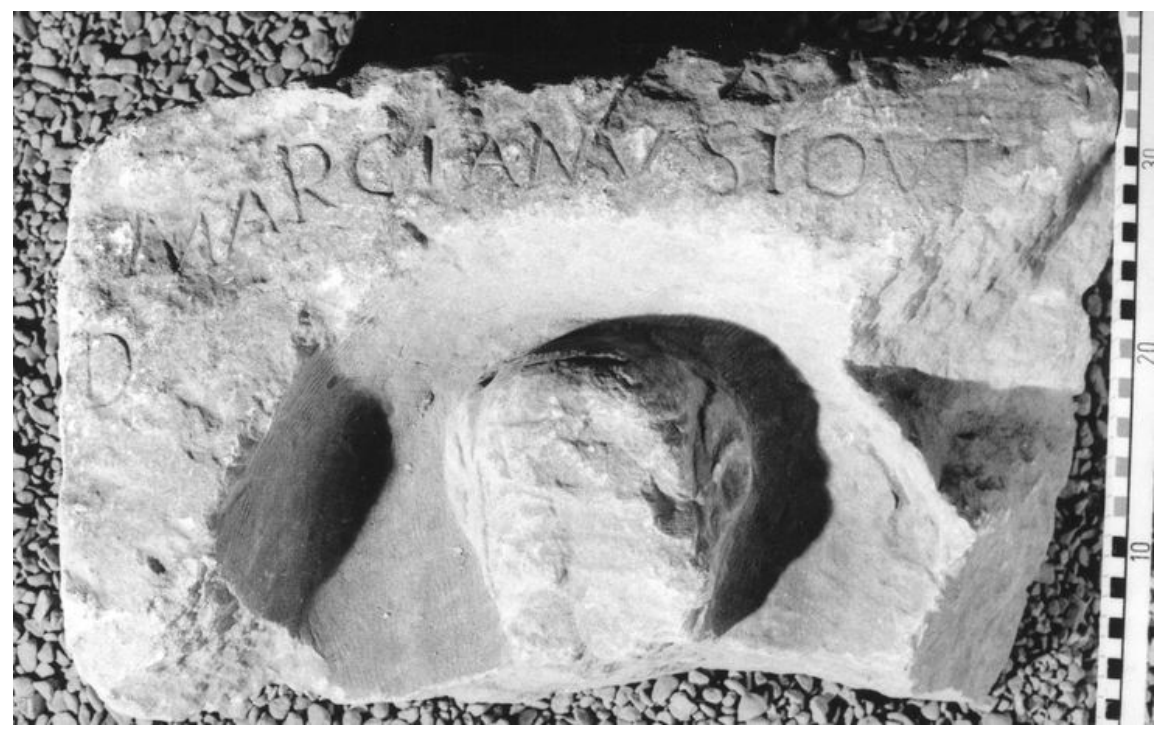

Abb.8: Grabinschrift aus Dijon/Dibio: CIL XIII 5535

(C) CIL XIII/2-Projekt, Universitäten Trier/Flensburg)

Damit folgt LE BOHEC grundsätzlich der Lesung im CIL. Dort werden die noch vorhandenen Buchstaben des Steins mit MARCIANVS IOVII angegeben. ${ }^{55}$ Entsprechend liest EsPÉRANDIEU den Namen Marcianus Iovi... (filius). ${ }^{56}$ Unter Berufung auf KAJANTO sieht LE BOHEC in Iovius einen lateinischen Namen. Allerdings kann KAJANTO für den Namen nur auf einige Belege aus der Spätantike verweisen, ${ }^{57}$ u.a. auf Iovius als Beiname Diocletians, der den Namen aufgrund seiner propagandierten Abstammung von Jupiter führte. ${ }^{58}$

Wecken die seltenen Belege aus dem Imperium für den Namen Iovius bereits Zweifel an der korrekten Lesung des Vaternamens auf dem vorliegenden Stein, zeigt eine nähere Betrachtung der Inschrift, dass diese Zweifel durchaus gerechtfertigt sind. Statt der Buchstabengruppe IOVI ist nämlich m.E. vielmehr TOVT zu lesen. Bei dem letzten, nur noch im oberen Bereich erhaltenen, Buchstaben der rechten Seite handelt es sich wahrscheinlich um ein I. Somit kann mit einiger Berechtigung Touti bzw. Touti [f(ilius)] gelesen werden.

Der keltische Name Toutus, der auf*touto- (= Stamm, Volk) zurückgeht, findet sich mehrfach im gallischen Raum. ${ }^{59}$ Der nächste Beleg stammt aus Metz/Divodurum. ${ }^{60}$ Eine Grabinschrift aus Augst/Augusta Raurica bietet den Namen Toutio. ${ }^{61}$ Und in dem Dijon/Dibio benachbarten Langres/Andemantunnum findet sich ein Beleg für das verwandte Toutonus. ${ }^{62}$

\footnotetext{
${ }^{55}$ CIL XIII 5535. S. ferner CIL XIII/5 36 (Index) („Iovii (an Iovi[n...]?“).

56 ESPÉRANDIEU 1911, 399. Nr.3492 (mit Photo).

${ }^{57}$ KAJANTO 1965, 212 mit nur einem konkreten Verweis auf Iobius(!), bezeugt durch eine Inschrift aus Africa aus dem Jahre 553 n. Chr.: AE 1956, 125 (Hippo Regius). S. ferner einen Nachweis aus der Moesia Superior: AE 1981, 734 (Lipjan). Das OPEL II führt darüber hinaus zwei Belege für Iovius als Gentilnomen: CIL III 10222 (Sremska Mitrovica/Sirmium; ein praefectus equitum Ituraeorum), XII 213 (Antibes/Antipolis).

${ }^{58}$ KAJANTO 1965, 212.

${ }^{59}$ Zum Namen s. OPEL IV 128, DelamarRe 2007, 184, 234.

${ }^{60}$ CIL XIII $11413=$ RIESE 1914, 388. Nr.3918.

${ }^{61}$ CIL XIII $5278=$ EDH Nr.HD076014.

${ }^{62}$ AE 1969/70, 427 = LE BOHEC 2003, 262. Nr.462 = EDH Nr.HD013879.
} 
VIII.

Aus Langres/Andemantunnum (dép. Haute-Marne), und damit ebenfalls aus dem Gebiet der Lingonen, stammt ein weiterer Grabstein (Höhe $155 \mathrm{~cm}$ - Breite $58 \mathrm{~cm}$ ) mit Darstellung des Verstorbenen in einer Giebelnische (Abb.9). Das Objekt aus Kalkstein fand sich (im vorletzten Jahrhundert?) in der Zitadelle von Langres. LE BOHEC datiert auch diesen Stein aufgrund der Formel $D$ (is) M(anibus) in das zweite Jahrhundert n. Chr. Die Inschrift des Steins, die bis auf die Buchstaben der ersten Zeile auf einem Schriftfeld in Form einer Tabula ansata steht, liest LE BOHEC wie folgt: ${ }^{63}$

$\begin{array}{ll} & \text { D(iis) } M(\text { anibus }) \\ & \text { April }<l>\text { is } \\ & \text { Montani (filii) } \\ & \text { Aprillan(us) } \\ 5 \quad & \text { p(onendum) c(uravit) }\end{array}$

Auch bei diesem Stein vertraut LE BOHec grundsätzlich auf die Lesung im CIL, wenngleich das CIL die Buchstaben der Zeile 2 mit APRILLIS angibt. Dem CIL folgt auch EsPÉRANDIEU, der jedoch in Zeile 2 den Namen Aprilis liest. ${ }^{64}$ Dagegen findet sich in allen genannten Publikationen übereinstimmend die Lesung des Namens $\operatorname{Aprillan}(u s)$ in Zeile 4.

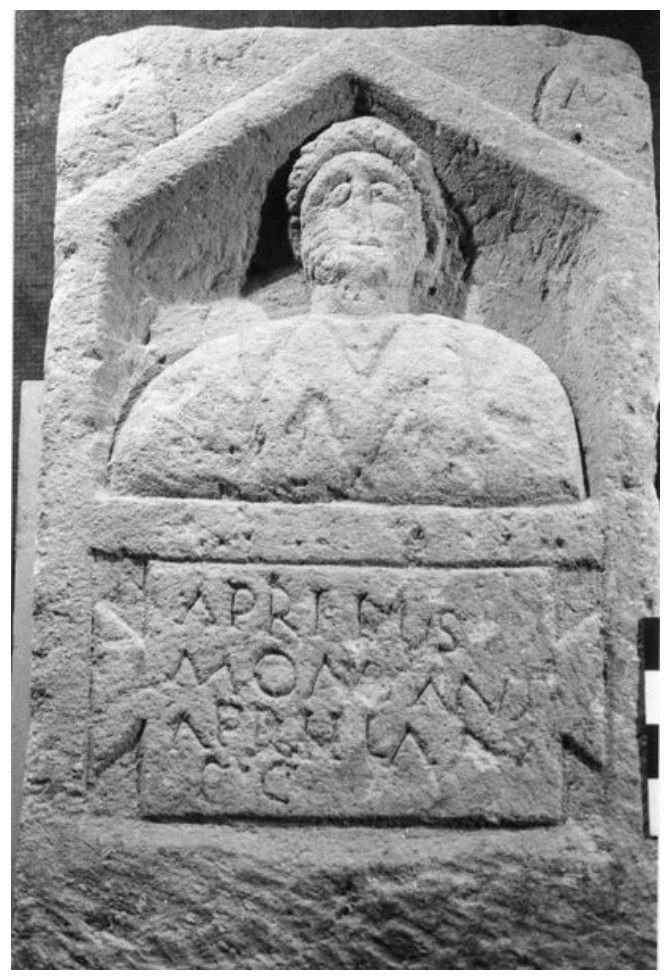

Abb.9: Grabinschrift aus Langres/Andemantunnum: CIL XIII 5716

(C) CIL XIII/2-Projekt, Universitäten Trier/Flensburg)

\footnotetext{
${ }^{63}$ LE BoHeC 2003, 237. Nr.418. Abb.189 (Photo). Vgl. auch die entsprechende Lesung der EDCS (Nr.10801128). - Der Stein befindet sich seit seiner Entdeckung im Musée de Langres.

${ }^{64}$ EsPÉRANDIEU 1911, 279. Nr.3240 (mit Photo).
} 
Eine korrekte Lesung des gesamten Textes wird durch die nachlässig bzw. nicht fachkundig eingeschlagenen Buchstaben sicher erschwert. Dennoch lässt sich in Zeile 2 mit Sicherheit der Name Aprillis mit geminiertem L und verkleinertem I entziffern. Dass der Dedikant der Inschrift den abgekürzten Namen Aprillanus trug, kann jedoch bezweifelt werden. M.E. ist in Zeile 3 der Name Aprilla zu lesen. Für Aprillanus lassen sich keine weiteren Belege nachweisen und Weiterbildungen auf -anus sind ohnehin nicht allzu verbreitet. ${ }^{65} \mathrm{Im}$ Gegensatz dazu finden sich für Aprilla, einen femininen Diminutiv des Namens Aper bzw. Apra, weitere Belege. Dabei stammt ein Nachweis auch aus Langres/Andemantunnum. ${ }^{66}$

Bei dem noch verbleibenden Buchstaben am Ende von Zeile 3 handelt es sich offenbar um ein ungewöhnlich lang gezogenes N. Vielleicht sollte hier ein $\mathrm{M}$ für $m$ (ater) stehen. Ebenso kann in der letzten Zeile, in der offenbar am ehesten $\mathrm{C} \mathrm{C} \mathrm{zu}$ lesen ist, wohl zum gängigen $\mathrm{P} C$ korrigiert werden, da sich für die Buchstaben $\mathrm{C} \mathrm{C}$ keine überzeugende Auflösung anbietet.

Somit lässt sich für die Grabinschrift aus Langres/Andemantunnum mit Vorsicht folgende Lesung vorschlagen:

\author{
$D$ (is) M(anibus) \\ Aprillis(!) \\ Montani (filii oder filius) \\ Aprilla ' $m{ }^{\top}$ (ater) \\ $5 \quad p^{\top}$ (onendum) $c$ (uravit)
}

IX.

Im Auftrag des Kurfürsten von Mainz publizierte der Benediktinerpater JoSEPH FUCHS in der zweiten Hälfte des 18. Jahrhunderts eine mehrbändige Abhandlung über die frühe Geschichte der Stadt Mainz. Im zweiten Band des Drucks aus dem Jahre 1772 findet sich eine bemerkenswerte römerzeitliche Grabinschrift aus MainzKastel/Castellum Mattiacorum, die im Jahre 1772 bereits verloren war (Abb.10). Laut FuCHS gehört der Stein zu einer Gruppe von acht weiteren Inschriften. ${ }^{67}$ Alle Steine ,sind [...] unter Kastell in der Gegend von St. Jörgen Kirchhofe, wo vor Zeiten die Clause oder Cluse und die Martinskirche gestanden ist, theils ausgegraben, theils aus den Mauern ausgebrochen worden." Über den Verbleib der Inschriften berichtet FuCHS: „Einige davon sind in den Fundamenten der jetzigen Pfarrkirche zu Kastell, und des Rathhauses vermauert worden, die anderen hat ein gewisser ...... Secretär von den Landleuten gekauft, und den Rhein hinab geschicket in den Jahren 1761 und $1762[\ldots]^{\text {“ }}$ Die Wiedergabe der Texte basiert laut FUCHS auf den Abschriften eines Geistlichen. Ausdrücklich betont FUCHS, dass er für die korrekte Abschrift der Texte keine Garantie geben kann. ${ }^{68}$

\footnotetext{
${ }^{65}$ KAJANTO 1965, 107-108.

${ }^{66}$ CIL XIII 5718 = LE BOHEC 2003, 238. Nr.420. Generell zum Namen Aprilla s. OPEL I ${ }^{2}$ 68, KAKoschKe 2008, 113. CN 257. - Zu Aprillanus s. KAJANTO 1965, 107-108, 219, SOLIN/SALMIES ${ }^{2}$ 294, OPEL I ${ }^{2}$ 68, KAKOSCHKE 2008, 113. CN 256 (jeweils mit dem Beleg aus Langres/ Andemantunnum).

${ }^{67}$ CIL XIII 7300, 7304-7306, 7308, 7309, 7312, 7313.

${ }^{68}$ FUCHS 1772, 172. - Zu FUCHS und seinen Schriften s. zuletzt M. J. KLEIN, MZ 102 (2007), 92-95.
} 


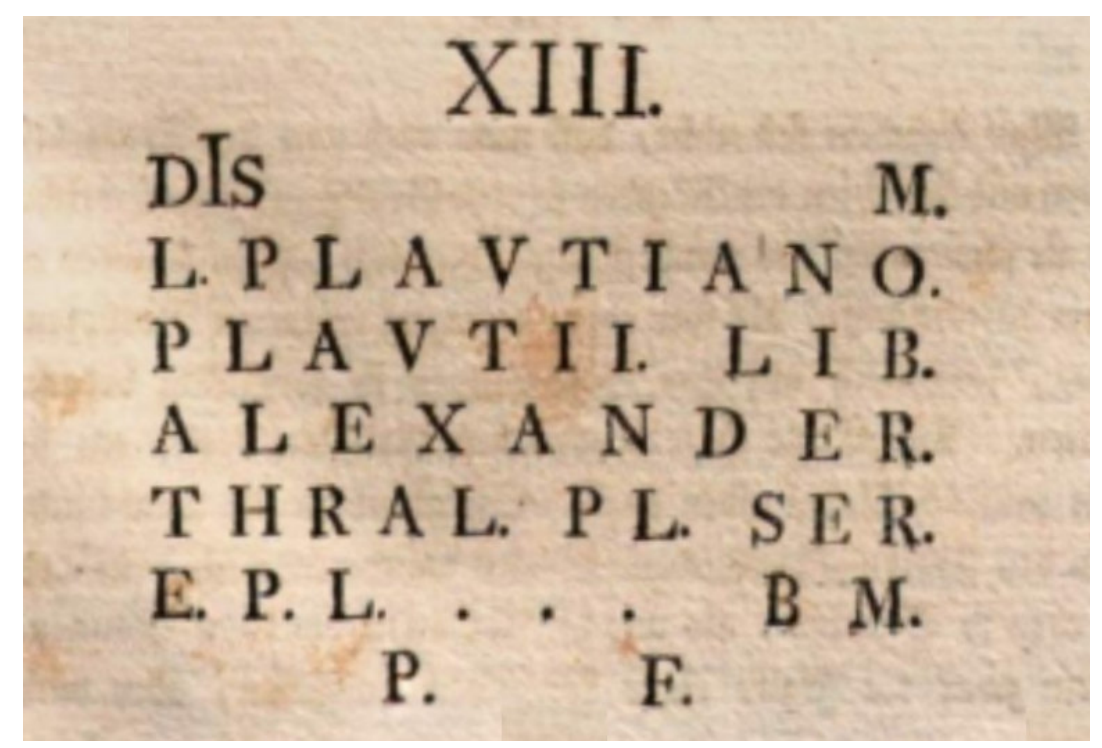

Abb.10: Grabinschrift aus Mainz-Kastel/Castellum Mattiacorum: CIL XIII 7310 (aus: FUCHS 1772, 175)

Die hier zu behandelnde Grabinschrift fand gemäß den Angaben bei FucHS Aufnahme im Inschriftencorpus BRAMBACHs ${ }^{69}$ und unter der Nummer 7310 auch Eingang in das CIL XIII. In der EDH wird die Inschrift mit der Bemerkung „Lesung zweifelhaft und Zuordnung der Namensbestandteile unsicher" wie folgt wiedergegeben: ${ }^{70}$

$\begin{array}{ll} & \text { Dis M(anibus) } \\ & \text { L(ucio) Plautiano } \\ & \text { Plautii lib(erto) } \\ & \text { Alexander } \\ & \text { Thral(---) Pl(auti) ser(vus) } \\ & \text { E(---) P(---) L(---) [---] b(ene) m(erenti) } \\ & p(---) f(---)\end{array}$

ZANGEMEISTER erblickt in der Inschrift eine Fälschung oder zumindest einen fehlerhaft überlieferten Text. So sollen die in der Inschrift genannten Namen laut ZANGEMEISTER auf die Namen historischer Persönlichkeiten zurückgehen. ZANGEMEISTER nennt in diesem Zusammenhang folgende Personen: den Prätorianerpräfekten C. Fulvius Plautianus, dessen Sohn C. Fulvius Plautus Hortensianus und den byzantinischen Arzt Alexander Trallianus. ${ }^{71}$

Andere Autoren, wie WIERSCHOWSKI und LAZZARO halten die Inschrift dagegen für authentisch. Dabei bleibt die Lesung des Textes jedoch umstritten. Laut FUCHS wurde die Inschrift von einem „Alexander von Thrallis des Plautius Knecht“ für einen „Lucius Plautianus des Plautius Freygelassenen“ gesetzt. ${ }^{72}$ Dieser Auffassung folgt weitgehend auch LAZZARO, der der Inschrift die Namen „L. Plautius Plautianus“(!) und „Alexander Thral(lianus)“ entnimmt. ${ }^{73}$ WIERSCHOWSKI erblickt in dem Verstorbenen dagegen einen „L. Plautianus Plauti(!) 1. Alexander“ aus

\footnotetext{
${ }^{69}$ BRAMBACH 1867, 248. Nr.1351.

${ }^{70}$ EDH Nr.HD080599. Vgl. auch die entsprechende Lesung in der EDCS (Nr.11001400).

${ }^{71}$ CIL XIII 7310. - S. auch Riese 1914, 404. Nr.4103 (,,vielleicht gefälscht“").

72 FUCHS 1772, 176.

${ }^{73}$ LAZZARO 1993, 210. Nr.231. Vgl. auch LAZZARO 1993, 539 (Index).
} 


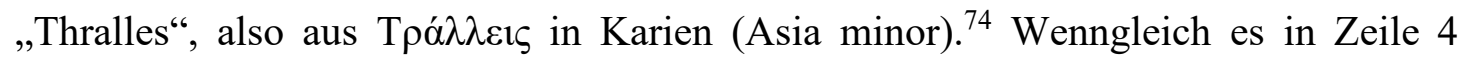
(wohl fehlerhaft) Alexander und nicht Alexandro heißt, erscheint die Auffassung WIERSCHOWKIs überzeugender. Schließlich wird der verstorbene libertus sicher ein Cognomen geführt haben, welches sein früherer Sklavenname war. Ferner darf man in THRAL am ehesten eine Herkunftsangabe erblicken. Zwar lassen sich auch Namen wie Trallianus, Trallus und Trallis aufführen, doch sind diese (im Westen) recht selten. ${ }^{75}$ Am Rande sei ferner auf einen Mann aus Köln/CCAA verwiesen, der die (latinisierte) Herkunftsangabe Trallianus gibt. ${ }^{76}$ Die Anwesenheit von Personen aus der kleinasiatischen Stadt im Rheingebiet ist demnach bezeugt.

$\mathrm{Ob}$ es sich bei dem überlieferten Dokument um einen antiken Text handelt, kann an dieser Stelle selbstverständlich nicht endgültig entschieden werden. Möglicherweise bietet die interessante Namensform des Verstorbenen jedoch einen bisher nicht berücksichtigten Anhaltspunkt. Offenbar trägt der Verstorbene ein Gentiliz mit der Endung -ianus. ${ }^{77}$ Diese eher seltenen Namen finden sich - wie die zuvor behandelte Inschrift aus Morken-Harff zeigt (s.o.) - in Niedergermanien sowie in Italien. Bei den Belegen aus dem ubischen Raum handelt es sich um neue Bildungen, um Pseudogentilnomina, die von einem Cognomen oder einem Nomen simplex abgeleitet wurden. In den Belegen aus dem italischen Raum darf man dagegen alte Gentilnomina erblicken. Gentilnomina auf -ianus sind darüber hinaus für den griechisch geprägten Kulturraum charakteristisch. Bei Solin/SALOMIES aufgeführte -ianusNamen wie z.B. Aurelianus, Claudianus, Fuscianus, Sallustianus, Sergianus oder Vettianus treten nur im Osten des Imperiums und fast immer auf griechischen Inschriften in Erscheinung. ${ }^{78}$ Der Verbreitungsschwerpunkt dieser -ianus-Namen liegt in Makedonien und in Kleinasien. ${ }^{79}$ Der in Mainz-Kastel/Castellum Mattiacorum verstorbene L. Plautianus Alexander stammt also aus einem Raum, in dem -ianus-Namen verbreitet waren.

Laut SALOMIES wurden die meisten -ianus-Namen aus der griechischen Reichshälfte aus gängigen römischen Gentilnomina auf -ius gebildet. So gehen die zuvor aufgeführten Namen auf die Gentilnomina Aurelius, Claudius, Fuscius, Sallustius, Sergius und Vettius zurück. Gentilnomina auf -ius konnten im Osten willkürlich durch entsprechende Namen auf -ianus ausgetauscht werden. SALOMIES verweist z.B. auf den Konsul des Jahres 175 n. Chr., L. Calpurnius Piso, der in einer Inschrift aus Phrygien das Gentiliz Calpurnianus führt. ${ }^{80}$ Ein Mann aus Lykaonien in

\footnotetext{
${ }^{74}$ Wierschowski 19, 136. Vgl. auch CIL XIII/5 16 (Index) (,"*L. Plautianus Plautii lib. Alexander“), 149 (Index) [,*T(h)ral(lianus)“], KAKOSCHKE 2002, 104. Nr.1.75 („Der Verstorbene trug wohl den Namen 'L. Plautianus Alexander' und war der libertus eines Plautius.“) und O. SCHIPP, BJb 213 (2013), 102, 111 [,... Lucius Plautianus Alexander aus Aydin (Tralleis) in Karien (wenn diese Lesart richtig ist) ...." $]$.

${ }^{75}$ Das OPEL IV führt nur einen Beleg für den femininen Namen Trallis in Oberitalien: CIL V $82=$ Pais 1888, Nr.7 (Pula/Pola). Die nächsten Belege finden sich in Rom: CIL VI 18843 (Trallianus und Trallis), 23220 (Trallis), AE 2005, 251 (Trallis).

${ }^{76}$ CIL XIII 8323 = EDH Nr.HD075880. - S. ferner die Grabinschrift CIL XIII 3684 = AE 1996, 1098 (Trier/Augusta Treverorum), die einen in Tralles verstorbenen (in Asia Trallis defuncto) centurio ex cohortis primae Hamiorum bezeugt.

${ }^{77}$ SOLIN/SALOMIES ${ }^{2}$ und das OPEL führen den Namen nicht auf. S. jedoch KAKOSCHKE 2006, 311-312. GN 962.

${ }^{78}$ Solin/SALOMIES ${ }^{2}$ 1994, 276-277. Eine Liste der -ianus-Namen aus dem Osten des Imperiums bietet auch SALOMIES 1984, 101-102.

${ }^{79}$ Grundsätzlich zu Gentilnomina auf -ianus s. SALOMIES 1984, 97-104, A. KAKOSCHKE, FeRA 38 (2019), 5-24.

${ }^{80}$ SALOMIES 1984, 103 mit dem Beleg IGR IV 521 (Dorylaion).
}

http://www.fera-journal.eu 
Kleinasien gibt den Namen G. Aponianos Sopatros an, während sein Vater $G$. Aponios Longos hieß. ${ }^{81}$

Im Hinblick auf die Inschrift aus Mainz-Kastel/Castellum Mattiacorum ist eine Inschrift aus Seferihisar/Teos in der Provinz Asia minor von besonderem Interesse. Der Text erwähnt die liberti eines C. Venuleius Flaccinus, die sich Venuleiani Dromo et Verna nennen. ${ }^{82}$ Ebenso wie der in Obergermanien verstorbene Freigelassene formten die liberti aus der östlichen Provinz ihr Gentiliz aus dem Gentiliz ihres Patrons bzw. sie ersetzten den -ius-Namen durch einen -ianus-Namen. Weitere Belege für eine entsprechende Namensübernahme bei Freigelassenen sind offenbar nicht bezeugt. ${ }^{83}$

Wenn es sich bei der Inschrift aus Mainz-Kastel/Castellum Mattiacorum um eine Fälschung handelt, war der Fälscher aus dem 18. Jahrhundert entweder ein sehr guter Onomastiker mit Kenntnissen in der Namensgebung im griechischsprachigen Raum oder er hat durch reinen Zufall einen überzeugenden mehrteiligen Namen für einen Freigelassenen aus Kleinasien konstruiert. Dabei ist Letzteres wohl nicht völlig auszuschließen, da der Sinn des Textes auch durch moderne Texteingriffe, durch die korrigierende Lesung Alexand ${ }^{\prime}{ }^{\prime}{ }^{\top}{ }^{\top}$ bzw. $T\{h\}$ ral(liano), erst geschaffen wird. Ferner ist noch darauf hinzuweisen, dass sich für die Namensformel „Pränomen + (Pseudo-) Gentiliz + Gentilnomen des Patrons im Genitiv (mit der Angabe libertus) + Cognomen" zumindest in den germanischen Provinzen keine weiteren Belege finden.

\section{Siglen}

1. N.

H. FINKE, Neue Inschriften, BRGK 17 (1927), 1-107, 198-231.

2. N.

H. Nesselhauf, Neue Inschriften aus dem römischen Germanien und den angrenzenden Gebieten, BRGK 27 (1937), 51-134.

3. N.

H. Nesselhauf / H. LIEB, Dritter Nachtrag zu CIL. XIII. Inschriften aus den germanischen Provinzen und dem Treverergebiet, BRGK 40 (1959), 120-229.

$\mathrm{AE}$

L'Année Épigraphique, Paris.

AKB

Archäologisches Korrespondenzblatt, Mainz.

$\mathrm{BJb}$

Bonner Jahrbuch, Bonn.

BRGK

Bericht der Römisch-Germanischen Kommission, Mainz.

CIL Corpus Inscriptionum Latinarum, Berlin 1863ff.

GFA

Göttinger Forum für Altertumswissenschaft, Göttingen. (www.gfa.gbv.de)

\footnotetext{
${ }^{81}$ SALOMIES 1984, 102 mit dem Beleg MAMA VIII 94.

${ }^{82}$ AE 1924, 45 = EDH Nr.HD026302.

${ }^{83}$ Nicht vergleichbar ist z.B. der Name des Steuereintreibers Donatus Caesaris Au[g(usti servus)] Salvianus (CIL XIII 5092 = EDH Nr.HD067879) aus Avenches/Aventicum. Der Sklave gehörte offenbar vormals zur familia des Kaisers Salvius Otho. Er formte seinen zweiten Namen, sein zweites Nomen simplex(!), wie allgemein üblich aus dem Gentiliz seines früherern Herrn, um seine alte Zugehörigkeit anzuzeigen.
} 
IGR

ILGN

MAMA

$\mathrm{MZ}$

OPEL I ${ }^{2}$ IV

SJ

ZPE

BRAMBACH 1867

DELAMARRE 2007

FUCHS 1772

ESPÉRANDIEU 1911

ESPÉRANDIEU 1931

FREIS ${ }^{2} 1994$

GOUBET 2015

GUTENBRUNNER 1936
Inscriptiones Graecae ad res Romanas pertinentes, Paris.

É. ESPERANDIEU, Inscriptions latines de Gaule (Narbonnaise), Paris 1929.

Monumenta Asiae Minoris Antiqua, London.

Mainzer Zeitschrift, Mainz.

Onomasticon Provinciarum Europae Latinarum I2-IV. Ex materia ab A. MócSY, R. FELDMANN, E. MARTON et M. SZILÁGYI collecta, hrsg. von B. LÖRINCZ u.a., Budapest-Wien 1999-2005.

Saalburg Jahrbuch, Mainz.

Zeitschrift für Papyrologie und Epigraphik, Bonn.

\section{Literaturverzeichnis}

W. BRAMBACH, Corpus Inscriptionum Rhenanarum, Elberfeld 1867.

X. Delamarre, Nomina Celtica antiqua selecta inscriptionum (Noms de personnes celtiques dans l'épigraphie classique), Paris 2007.

J. FuCHS, Alte Geschichte von Mainz. Aus den ältesten und ersten Zeiten, von dem Anfange dieser Hauptstadt unter dem Kaiser Augustus bis zu Ende des siebenden Jahrhundert. Bd.2. Enthält die Zeiten vom 98. bis zu dem 180. Jahr, Mainz 1772.

É. ESPÉRANDIEU, Recueil général des bas-reliefs, statues et bustes de la Gaule romaine 4, Paris 1911.

É. ESPÉRANDIEU, Recueil général des bas-reliefs, statues et bustes de la Germanie romaine. Complément du Recueil général des bas-reliefs, statues et bustes de la Gaule romaine, Paris-Bruxelles 1931.

H. FREIS, Historische Inschriften zur römischen Kaiserzeit von Augustus bis Konstantin. 2., durchges. Aufl., Texte der Forschung 49, Darmstadt 1994.

F. GOUBET / F. JODRY / N. MEYER / N. WeISS, Au „Grès“ du Temps. Collection lapidaires celtes et gallo-romaines du Musée archéologique de Saverne, Saverne 2015.

S. GutENBRUNNER, Die germanischen Götternamen der antiken Inschriften, Rheinische Beiträge und Hülfsbücher zur germanischen Philologie und Volkskunde 24, Halle (Saale) 1936. 
HERZ 1989

JOUBEAUX 1989

KAJANTO 1965

KAKOSCHKE 2002

KAKOSCHKE 2006

KAKOSCHKE 2007

KAKOSCHKE 2008

KoLBE 1960

KUHN 1978

LAZZARO 1993

LE BOHEC 2003

MEIER-ARENDT 1983

PAIS 1888
P. HERZ, Altbürger und Neubürger. Bemerkungen zu einer Inschrift aus dem römischen Heddernheim, AKB 19 (1989), 159-167.

H. JoubeauX, Un type particulier de monuments funéraires: les ,pyramidions“ des nécropoles galloromaines de Dijon, Gallia 46 (1989), 213-244.

I. Kajanto, The Latin Cognomina, Societas Scientiarum Fennica. Commentationes Humanarum Litterarum 36.2, Helsinki-Helsingfors 1965.

A. KAKOSCHKE, Ortsfremde in den römischen Provinzen Germania inferior und Germania superior. Eine Untersuchung zur Mobilität in den germanischen Provinzen anhand der Inschriften des 1. bis 3. Jahrhunderts n. Chr., Osnabrücker Forschungen zu Altertum und Antike-Rezeption 5, Möhnesee 2002.

A. KakoschKe, Die Personennamen in den zwei germanischen Provinzen. Ein Katalog. Bd.1: Gentilnomina ABILIUS-VOLUSIUS, Rahden/Westf. 2006.

A. KakoschKe, Die Personennamen in den zwei germanischen Provinzen. Ein Katalog. Bd.2,1: Cognomina ABAIUS-LYSIAS, Rahden/Westf. 2007.

A. KAKoschKe, Die Personennamen in den zwei germanischen Provinzen. Ein Katalog. Bd.2,2: Cognomina MACCAUS-ZYASCELIS, Rahden/Westf. 2008.

H.-G. KolBE, Die neuen Matroneninschriften von Morken-Harff, BJb 160 (1960), 50-124.

H. KuHN, Kleine Schriften. Aufsätze und Rezensionen aus den Gebieten der germanischen und nordischen Sprach-, Literatur- und Kulturgeschichte 4, Berlin-New York 1978.

L. LAZZARO, Esclaves et affranchis. En Belgique et Germanies Romaines d'après les sources épigraphiques, Annales Littéraires de 1'Université de Besançon 430, Paris 1993.

Y. LE BOHEC, Inscriptions de la cité des Lingons. Inscriptions sur pierre, Inscriptiones latinae Galliae Belgicae 1. Lingones, Paris 2003.

W. MEIER-ARENDT, Römische Steindenkmäler aus Frankfurt am Main. Auswahlkatalog, Archäologische Reihe 1, Frankfurt am Main 1983.

E. PAIS, Corporis inscriptionum latinarum supplementa Italica consilio et auctoritate academiae regiae Lynceorum edita I. Additamenta ad vol. V. Galliae Cisalpinae, Rom 1888.

http://www.fera-journal.eu 
RAEPSAET-CHARLIER 2011 M.-TH. RAEPSAET-CHARLIER, Les noms germaniques: adaptation et latinisation de l'onomastique en Gaule Belgique et Germanie inférieure, in: M. DONDIN-PAYRE (Hrsg.), Les noms de personnes dans l'Empire romain, Paris 2011, 203-234.

REICHERT 1987-1990

H. REICHERT, Lexikon der altgermanischen Namen. 2 Teile, Thesaurus Palaeogermanicus 1, Wien 1987-1990.

RIESE 1914

RÖSGER 1996

A. RIESE, Das rheinische Germanien in den antiken Inschriften, Leipzig-Berlin 1914.

A. RÖSGER, Römer, Ubier, Franken. Rödingen von 53 v. Chr. bis ca. 450 n. Chr., in: Zur Geschichte von Rödingen, Höllen und Bettenhoven. 846 - 1996, Redaktion: P. BITTNER u.a., Rödingen 1996, 9-24.

SALOMIES 1984

O. SALOMIES, Beiträge zur römischen Namenkunde, Arctos 18 (1984), 93-104.

SCHILLINGER-HÄFELE 1982 U. SCHILLINGER-HÄFELE, Lateinische Inschriften. Quellen für die Geschichte des römischen Reiches, Limesmuseum Aalen. Kleine Schriften zur Kenntnis der römischen Besetzungsgeschichte Südwestdeutschlands 23, Waiblingen 1982.

SCHOPPA $^{2} 1965$

H. SCHOPPA, Sammlung Nassauischer Altertümer Wiesbaden. Städtisches Museum: Der römische Steinsaal. 2., unveränderte Aufl., Schriften des Städtischen Museums Wiesbaden 3, Wiesbaden 1965.

SCHULZE 1904

W. Schulze, Zur Geschichte lateinischer Eigennamen, Abhdlg. der Königl. Ges. der Wiss. zu Göttingen. Phil.Hist. K1. N. F. 5, Göttingen 1904 (Nachdr. Berlin-Zürich-Dublin 1966).

Solin/SAlOMIES ${ }^{2} 1994$ H. Solin / O. SAlOMIES, Repertorium nominum gentilium et cognominum Latinorum. 2. Aufl. Editio nova addendis corrigendisque augmentata, Alpha Omega. Reihe A. Lexika - Indizes - Konkordanzen zur klassischen Philologie 80, Hildesheim-Zürich-New York 1994.

STEINER 1851

J. W. CH. SteIner, Codex inscriptionum romanarum Danubii et Rheni. Bd.2. Inscriptiones Germaniae primae et Germaniae secundae, Seligenstadt-Groß-SteinheimDarmstadt 1851.

SPICKERMANN 1994

W. SPICKERMANN, „Mulieres ex Voto“. Untersuchungen zur Götterverehrung von Frauen im römischen Gallien, Germanien und Rätien (1.-3. Jahrhundert n. Chr.), Bochumer historische Studien. Alte Geschichte 12, Bochum 1994. 
STUART 1977

WEISGERBER 1968

WEISGERBER 1969

WHATMOUGH 1969

$\mathrm{EDH}$

EDR

LUPA

EDCS

P. STUART, Een Romeins grafveld uit de eerste eeuw te Nijmegen. Onversierde terra sigillata en gewoon aardwerk, Beschrijving van de verzamelingen in het Rijksmuseum G. M. Kam te Nijmegen 8, Leiden 1977.

J. L. WeISGERBER, Die Namen der Ubier, Wissenschaftliche Abhandlungen der Arbeitsgemeinschaft für Forschung des Landes Nordrhein-Westfalen 34, Köln-Opladen 1968.

J. L. WeIsGerber, Rhenania Germano-Celtica. Gesammelte Abhandlungen. Dem Autor zum siebzigsten Geburtstag am 25. Februar 1969, hrsg. von J. KNOBLAUCH und R. SCHÜTZEICHEL, Bonn 1969.

J. Whatmough, The dialects of ancient Gaul, (Mikrofilm) Cambridge/Massachusetts 1949ff. (Nachdr. Cambridge/Massachusetts 1970).

\section{Elektronische Datenbanken}

Epigraphik-Datenbank Clauss/Slaby (Frankfurt), Leitung: M. CLAuss u.a. (Johann Wolfgang Goethe-Universität Frankfurt am Main / Katholische Universität Eichstätt-Ingolstadt / Universität Zürich), Stand: 01.12.2019 (www.manfredclauss.de).

Epigraphische Datenbank Heidelberg, Leitung: $\mathrm{CH}$. WITSCHEL (Ruprecht-Karls-Universität Heidelberg), Stand: 01.12.2019 (www.uni-heidelberg.de/institute/ sonst/adw/edh).

Epigraphic Database Roma, Leitung: G. CAMODECA, S. ORLANDI (Università di Roma - La Sapienza), Stand: 01.12.2019 (www.edr-edr.it).

UBI ERAT LUPA, Bilddatenbank zu antiken Steindenkmälern (Universität Salzburg), Leitung: F. und O. HARL (Universität Wien), Stand: 01.12.2019 (www.ubi-eratlupa.org).

\section{Kontakt zum Autor:}

Dr. Andreas Kakoschke

Nelly-Sachs-Weg 1

D-49191 Belm

E-Mail: andreaskakoschke@hotmail.de 